\title{
Systematic genomic identification of colorectal cancer genes delineating advanced from early clinical stage and metastasis
}

\author{
HoJoon Lee ${ }^{1 \dagger}$, Patrick Flaherty ${ }^{2 \dagger}$ and Hanlee P Ji ${ }^{1,3^{*}}$
}

\begin{abstract}
Background: Colorectal cancer is the third leading cause of cancer deaths in the United States. The initial assessment of colorectal cancer involves clinical staging that takes into account the extent of primary tumor invasion, determining the number of lymph nodes with metastatic cancer and the identification of metastatic sites in other organs. Advanced clinical stage indicates metastatic cancer, either in regional lymph nodes or in distant organs. While the genomic and genetic basis of colorectal cancer has been elucidated to some degree, less is known about the identity of specific cancer genes that are associated with advanced clinical stage and metastasis.

Methods: We compiled multiple genomic data types (mutations, copy number alterations, gene expression and methylation status) as well as clinical meta-data from The Cancer Genome Atlas (TCGA). We used an elastic-net regularized regression method on the combined genomic data to identify genetic aberrations and their associated cancer genes that are indicators of clinical stage. We ranked candidate genes by their regression coefficient and level of support from multiple assay modalities.
\end{abstract}

Results: A fit of the elastic-net regularized regression to 197 samples and integrated analysis of four genomic platforms identified the set of top gene predictors of advanced clinical stage, including: WRN, SYK, DDX5 and ADRA2C. These genetic features were identified robustly in bootstrap resampling analysis.

Conclusions: We conducted an analysis integrating multiple genomic features including mutations, copy number alterations, gene expression and methylation. This integrated approach in which one considers all of these genomic features performs better than any individual genomic assay. We identified multiple genes that robustly delineate advanced clinical stage, suggesting their possible role in colorectal cancer metastatic progression.

Keywords: Colorectal cancer, Genomics, Genetics, Clinical stage, Metastasis

\section{Background}

Colorectal cancer (CRC) is projected to be the 3rd leading cause of cancer deaths in the United States in 2013 with the mortality primarily a result of metastatic disease [1]. Identifying the genetic and genomic basis of CRC has significant clinical implications. Our understanding of CRC requires identification of the critical "driver" genes that are fundamentally important for CRC development unlike

\footnotetext{
* Correspondence: genomics_ji@stanford.edu

${ }^{\dagger}$ Equal contributors

'Division of Oncology, Stanford University School of Medicine, Stanford, CA 94305, USA

${ }^{3}$ Stanford Genome Technology Center, Stanford University, Palo Alto, CA 94304, USA

Full list of author information is available at the end of the article
}

"passenger" genetic aberrations that have no functional relevance to cancer biology [2]. Previous genetic and genomic studies of CRC have identified many of the critical drivers that are important to CRC development [3-7]. For example, the cancer genes APC, KRAS and TP53 have a high frequency of genetic aberrations in CRC and are known to play an essential role in CRC development [8]. A number of other cancer genes have been identified in CRC and cluster in several biological pathways including those responsible for Wnt signaling [9], RAS/RAF pathway [10] and transforming growth factor $\beta$ (TGF- $\beta$ ) signaling [11]. While the cancer genes directly responsible for CRC development have been characterized, less is known

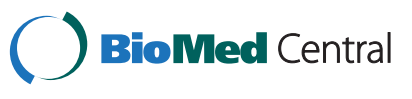

(c) 2013 Lee et al.; licensee BioMed Central Ltd. This is an Open Access article distributed under the terms of the Creative Commons Attribution License (http://creativecommons.org/licenses/by/2.0), which permits unrestricted use, distribution, and reproduction in any medium, provided the original work is properly cited. The Creative Commons Public Domain Dedication waiver (http://creativecommons.org/publicdomain/zero/1.0/) applies to the data made available in this article, unless otherwise stated. 
about which cancer genes delineate advanced versus early stage CRC.

Currently, the metastatic status of CRC is assessed via clinical staging which dictates the choice of therapy and remains the best prognostic indicator for individual CRC patients [12]. Clinical stage is determined by the TNM criteria, where $\mathrm{T}$ is assigned by extent of tumor invasion, $\mathrm{N}$ represents the number of lymph nodes with metastatic cancer and $\mathrm{M}$ represents the presence of metastatic cancer in other organs outside of the colon and lymph nodes. Advanced clinical stage either reflects metastatic cancer spread to the regional lymph nodes around the colon as in stage III or spread to organs outside of the colon or rectum as in stage IV. Advanced (stages III or IV) CRC has a significantly worse prognosis compared to early stage (stages I or II) that is generally considered curable. With the advent of genomic cancer medicine, there is increasing interest in identifying the specific CRC genetic aberrations and related cancer genes that define advanced clinical stage. Identification of these genetic aberrations and their corresponding cancer genes may illuminate the underlying genetics of advanced clinical stage CRC as well as have relevance in the prognostic assessment.

A recent large-scale study by the Cancer Genome Atlas (TCGA) is the most comprehensive CRC genomic survey to date [13]. The TCGA CRC project relied on a combination of next generation sequencing and microarray genomic platforms to characterize different CRC genetic aberration features and the individual affected genes. This project also provides clinical information about the metastatic status of individual patients via clinical stage information. The breadth of the TCGA genomic data sets provides a unique opportunity to consider different categories of genetic aberrations at individual gene resolution that other genomic studies have not considered [14-16].

Relying on the TCGA CRC data, we conducted a supervised analysis, integrating all of the multiple classes of available genomic feature data. The integrated data set included i) somatic mutations, ii) copy number alterations, iii) gene expression changes and iv) methylation. Our analysis uses elastic-net regression to estimate an optimal multiple linear regression of the clinical outcome on the space of genomic features. We analyzed this integrated genomic data set against clinical stage to delineate genes associated with advanced CRC.

Our study is unique and has specific strengths in many aspects compared to previous studies. Most importantly, with our integrated analysis method, we considered a full range of cancer genetic aberrations, otherwise described as genomic features. We identified specific cancer genes associated with advanced clinical stage; some of these genes have not been reported as being associated with cancer progression. The results of our analysis can be queried directly through a website (http://genomeportal. stanford.edu/tcga-crc).

\section{Methods \\ TCGA CRC genomic data}

Genomic data was obtained from the Broad Firehose (http://gdac.broadinstitute.org) which is one of the Genome Data Analysis (GDACs) for TCGA project. The data files from January 2013 analysis/standardization run of colorectal (COADREAD) cancer includes five genomics assays for each sample: DNA copy number variation, mRNA expression level by microarray/RNASeq, somatic mutations by whole exome sequencing, DNA methylation, and expression level of miRNA by RNASeq. MicroRNA data was analyzed separately in our analysis because the frequency of missing data is relatively high and the general ambiguity in regards to identifying the specific gene targets subject to expression changes.

Clinical information of the samples was obtained from the Broad Firehose and UCSC cancer genome browser [17]. The availability of clinical parameter data for each sample was highly variable; therefore, we focused on those parameters that had the most complete annotation among the largest number of samples. We selected two major clinical parameters for elastic-net analysis: microsatellite instability (MSI), a molecular CRC feature associated with loss of DNA mismatch repair and clinical stage information. We also examined the individual variables of clinical stage via the TMN criteria: i) $\mathrm{T}$ is for extent of primary tumor invasiveness; ii) $\mathrm{N}$ derived from the number of cancer positive lymph nodes; iii) $M$ is an indicator of metastasis in other organs beyond the primary site. These clinical outcomes were converted into ordinal values and used for subsequent elastic-net regression analysis (Additional file 1: Table S1).

We sought to differentiate between driver genetic aberrations in known and putative cancer genes versus passenger events in genes not related to cancer development. To eliminate non-contributing passengers, we relied on cancer genomic data resources cataloging known and putative cancer gene. These genes were identified from large-scale studies and curation of the scientific literature. We chose the genes for inclusion in our initial set using two data sources: Catalogue of Somatic Mutations in Cancer (COSMIC) and TCGA. We chose COSMIC because it has been both curated and validated. However, we were concerned that by imposing such high standards on genes that make it into the initial set, we might miss out on genes that are important, but not yet validated. To broaden the scope of our study and improved our identification of clinically relevant cancer genes, we added genes sets obtained from the TCGA project as part of their discovery studies. 
Overall, the COSMIC database contains 484 genes that have been shown to be associated with cancer development and thus are established or candidate cancer genes [8]. The TCGA CRC analysis identified a large number of known and putative cancer genes including: $32 \mathrm{mu}-$ tated genes, 353 genes with copy number changes, a 30 genes expression signature associated with tumor aggressiveness and a 344 genes methylation signature [13]. In addition, we included 20 genes known to be frequently mutated in colorectal cancer $[18,19]$. This resulted in a set of 1,192 known or putative cancer genes where there was significant genomic and literature information supporting their role in cancer (Additional file 2: Table S2).

\section{Data pre-processing and normalization}

We conducted our analysis with Matlab (Mathworks, Natick, MA) and we provide the individual scripts used (Additional file 3). We used a multi-step procedure to update, normalize and filter the gene meta-data. First, we reviewed every gene symbol in all genomic data to the HUGO Gene Nomenclature (HGNC) [20]. According to the HGNC gene designation (March 2013), 55 gene symbols were not current and 15 genes were not protein coding genes. In total, 1,122 genes were in the candidate list after eliminating these 70 genes.

From the array data, we eliminated any features that had missing measurements for $3 \%$ or more samples. We imputed remaining missing measurements with the median across samples for each feature. For instance, there were 183 methylation array features lacked any values in 6 or more samples ( $\geq 3 \%$ of 197 samples) for clinical stage analysis. The imputation occurred with 26 features in methylation data and 6 features in mRNA microarray data.

Since mRNA levels were measured using both microarray and sequencing technology platforms, we combined these measurements for each gene using principal component analysis (PCA) [21]. PCA performs singular value decomposition on the probes by platform data matrix to yield factor weights for each platform. These weights were used in a linear combination to produce a single value for each gene,

$$
\begin{aligned}
\text { mRNA-eigen }= & 0.8972 \times \text { mRNA-Array }+0.4417 \\
& \times \text { mRNA-Seq }
\end{aligned}
$$

where mRNA-array is the microarray measurement in log-fold change and RNA-Seq is the sequencing measurement in $\log (\mathrm{RPKM})$ for a particular gene. The mRNA-eigen value preserves on average $68.9 \%$ of the variance in the original data. Additional file 4: Figure S1) shows a scatter plot of mRNA measurements from both platforms and the principal eigenvector projection of the data.

We excluded CRC samples that were not measured in all genomic platforms. This filtration step retained 200 of the initial total 585 samples in the data set. Subsequently, we excluded the samples without clinical information (Additional file 5: Table S3). All 200 samples have clinical information about $\mathrm{N}$ status. However, there were several samples missing some clinical information; 3 samples missing clinical stage, 1 sample missing T status, 2 samples missing $M$ status and 2 samples missing MSI status.

After pre-processing of data, we normalized the scale of each feature. Briefly, each genomic feature was normalized by the standard deviation of each gene's measurement plus 10 percentile of the global standard deviations in each assay. This standard deviation correction factor is standard in microarray analysis [22] and minimizes the risk of generating outliers due to normalization. The normalization is,

$$
\hat{\mathrm{g}}(\mathrm{i}, \mathrm{j})=\frac{\mathrm{g}(\mathrm{i}, \mathrm{j})}{\operatorname{sd}(\mathrm{g}(\mathrm{i}))+s d_{10}(\mathrm{~g})}
$$

where $g(i, j)$ is the value for feature $i$ in sample $j, s d(g$ (i)) is the standard deviation across samples for feature $\mathrm{i}, \mathrm{sd}_{10}(\mathrm{~g})$ is the 10 -percentile value of standard deviations across features and $\hat{g}(i, j)$ is the normalized feature value.

To prepare the final data set ready for analysis with the elastic-net, we simply combined the four genomic data sets (copy number, mutation, methylation, mRNAeigen) into a single matrix representing 197 samples for clinical stage analysis. The data is available at our web portal (http://genomeportal.stanford.edu/tcga-crc/pages/ datainformation).

\section{Elastic-net analysis}

We used elastic-net regression to estimate an optimal multiple linear regression of the clinical outcome on the space of genomic features [23]. The elastic-net algorithm simultaneously performs linear regression to learn the coefficient weights associated with each genomic feature while limiting the number of predictors in the model to ensure the model is general. It performs well on independent data that was excluded from the analysis of the original primary data set. We used 10 -fold crossvalidation to identify the value of the regularization parameter that minimized the average mean-squared error on this held-out test set. An additional tuning parameter taking values between zero and one controls the inclusion of correlated predictors. The standard 'Least Absolute Shrinkage and Selection Operator' (LASSO), which is the prototype algorithm of elastic net, sets this value 
to zero to minimize the inclusion of correlated predictors; we set the value to the mid-point of 0.5 . The resulting coefficients from the regularized regression were used to rank genes by their association with clinical features. It is important to note that the features identified by this procedure operate as a panel. While each individual feature may not predict the outcome well, in combination the prediction accuracy is improved as several other studies have shown this in various goal of using genomic data [24-29].

\section{Ranking scheme}

In the primary results of elastic-net, a gene can appear at most four times when all four types of genomic data support the gene. The rank of each genomic feature is determined by its absolute value of regression coefficient in descending order. Our scoring scheme by ranks gives priority to genes in the top ranks or multiple ranks by two-step calculations. First, we calculated the unit score to weight the rank proportionally;

$$
\text { Unit point }=1,000 \div \sum_{r=1}^{n} \mathrm{r}
$$

where $\mathrm{r}$ is the rank and $\mathrm{n}$ is the total number of ranks in the result from elastic-net analysis. Second, the score of top ranked genomic feature will be $\mathrm{n}$ times higher than the score of the bottom ranked genomic feature;

$$
\text { Score of } g(\mathrm{i})=(\text { unit point }) \times(n-\mathrm{r}(\mathrm{i})+1)
$$

where $g(i)$ is the genomic feature, $r(i)$ is the rank of the genomic feature of $\mathrm{i}$, and $\mathrm{n}$ is the total number of ranks in the list. Finally, the score of a gene will be the sum of scores of genomic features from the gene. Therefore, a gene that has a highly ranked genomic feature and/or has multiple genomic features will have a higher score in overall.

\section{Assessment of profile robustness to variations in training data}

Nonparametric bootstrap resampling was used to assess the robustness of the set of top ranked genes to changes in the training data as has been previously validated [30]. The complete data set was resampled with replacement 3,000 times and the elastic-net regression was recomputed for each bootstrap data set. Additional file 6: Table S4 details the count of the number of times a feature was selected by the elastic-net regression. Features that are consistently selected by the bootstrap regression have high rank and low variance in the rank are robust to variations in the training data set.

\section{Web implementation}

To facilitate access to our analysis results, we provide a website that can be queried: http://genomeportal.stanford. edu/tcga-crc/. The TCGA COAD Resource runs on a 2x2.27GHz Quad Core Intel Xeon E5520 server, with 24GB memory, and Ubuntu 9.10 operating system. The web application is implemented in Ruby on Rails 4.0, running under Apache2 and Passenger 4. The underlying database is MySQL 5.1.7, which is hosted on a separate database server. Query and data download is via any current web browser. Recommended browsers and versions are: Chrome 28.0+, Internet Explorer 8.0+, Firefox $22.0+$, Safari $5.0+$.

\section{Results}

\section{Cancer genome atlas data for CRC}

The TCGA CRC genomic data was obtained from the Broad Institute where the Tier 3 data (gene level calls that have been fully processed) are archived. Genomic data files from January 2013 analysis of colon and rectal cancer included five genomics assays for each sample: DNA copy number variation by single nucleotide polymorphism (SNP) array analysis, gene expression levels by microarray/RNASeq, somatic mutations by whole exome sequencing, DNA methylation via arrays and expression level of micro-RNA by RNASeq. These genomic data sets were integrated into a single data matrix for analysis (see Methods). Micro-RNA (miRNA) data was analyzed separately because the frequency of missing data is relatively high and the general ambiguity in regards to identifying the specific gene targets subject to expression changes.

We used a gene-centric approach for this study. For each gene, there were four genomic features represented by i) mutations, ii) copy number variation (CNV), iii) gene expression and iv) methylation status. We selected a candidate set of 1,185 known or putative cancer genes for our analysis. Using this approach i) reduces the issue of passenger events of no genetic significance, ii) provides an improved regression analysis and iii) facilitates interpretation of results according to their potential role in biological processes related to cancer. The genomic data of the selected genes was downloaded, preprocessed and integrated into a single data matrix for elastic-net analysis (Figure 1). As a normalization step to facilitate our study, we rescaled the features by their standard deviation across samples. This step improved the robustness of the analysis. This single combined matrix has genomic values of genes (independent variables) and the clinical table contains specific clinical parameters such as microsatellite instability (MSI) status and clinical stage.

\section{Application of elastic net regression to integrated genomic features}

We used elastic-net regression to estimate an optimal multiple linear regression of the clinical outcome on 


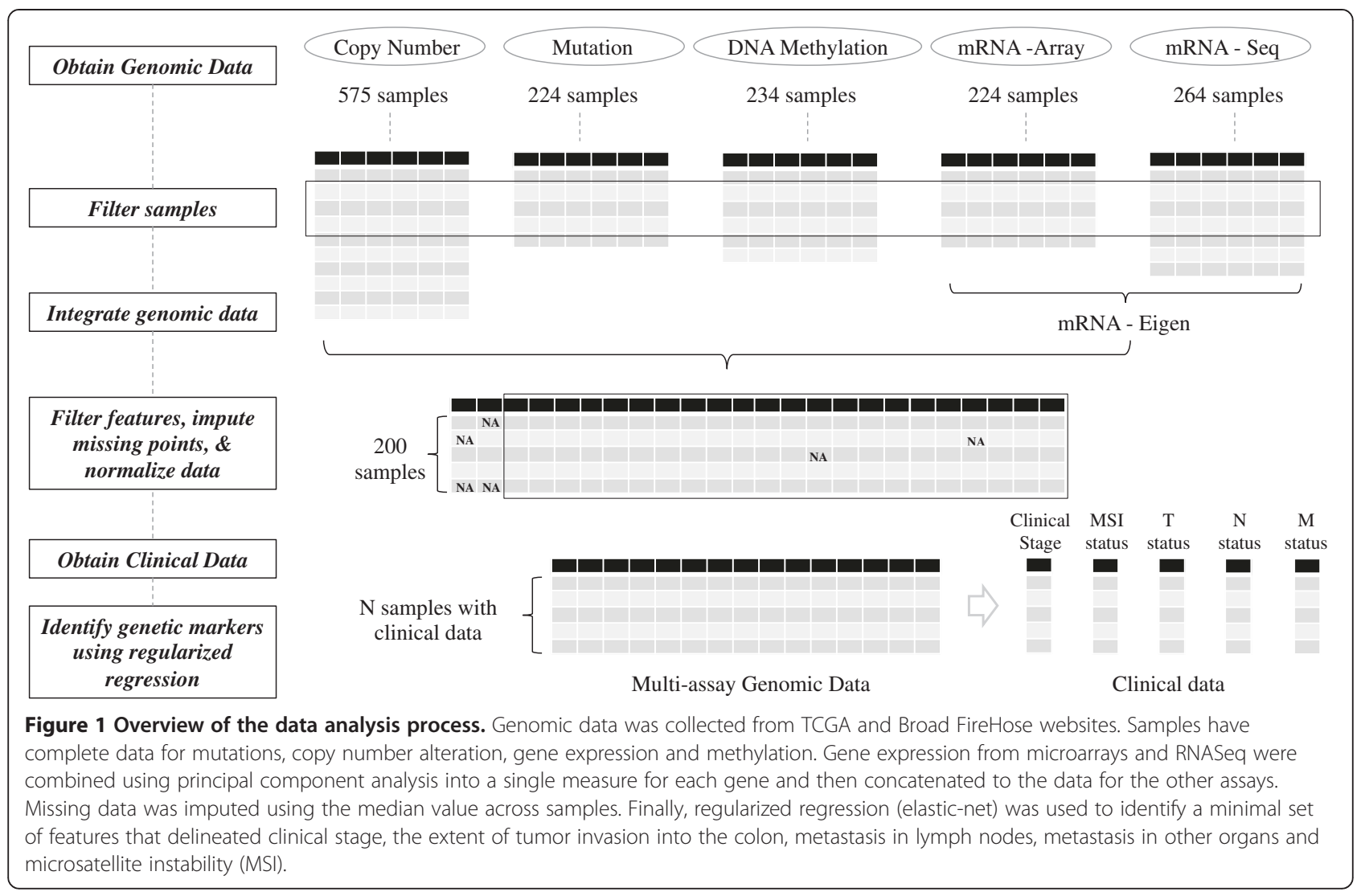

the integrated data set of all genomic features [23]. The elastic-net algorithm performs linear regression to learn the coefficient weights associated with genomic features simultaneously. Each coefficient in the elastic-net regression measures the partial correlation between the predictor feature (e.g. CNV, gene expression, methylation level or mutation status) and the outcome (e.g. clinical stage).

In general, a partial correlation is a measure of association between two variables after controlling for other variables - in this case the other variables are the other predictors in the regression (Figure 2). A positive coefficient - "direct association" - indicates that as the level of the predictor increases, the outcome increases after controlling for all other significant features. We indicate a direct association as a " $\uparrow$ ". Likewise, a negative coefficient - "inverse association" - means that as the predictor decreases, the outcome increases after controlling for other predictors (Figure 2). We indicate an inverse association as a " $\downarrow$ ".

Figure 2 shows comparison of lower versus higher advanced stage in direct/inverse association. The individual dots in each Case graph (Examples) represent stage I, II, III and IV respectively in order. The $\mathrm{X}$ axis represents clinical stages I through IV while Y axis represents genomic changes. Dotted lines imply normal status. Extra attention is required for Cases $\mathrm{C}$ and $\mathrm{F}$. Citing an example, Case $\mathrm{C}$ denotes that a reduced copy number such as might occur with a genomic deletion is more frequently observed in early stage compared to advanced stage CRC. Citing the reverse scenario, in Case F the amplification and thus increased gene copy number is observed in early stage while no copy number changes are noted in advanced stage CRC.

The resulting coefficients from the regularized regression were used to rank genomic features by their association with clinical features. The score of the genetic features was determined proportionally to their ranks and the score of each gene is the sum of all scores of its selected features (see Methods). This scoring scheme prioritizes genes that are supported by multiple genomic features and/or a highly ranked genomic feature. It is important to note that the features identified by this procedure operate as a panel. Several other genomic analysis studies have demonstrated that while an individual genomic feature may not predict the outcome, combining multiple genomic features improves the prediction accuracy [24-29].

\section{The performance of elastic-net}

To evaluate the utility and performance of elastic-net regularized regression for integrative genomic analysis, 


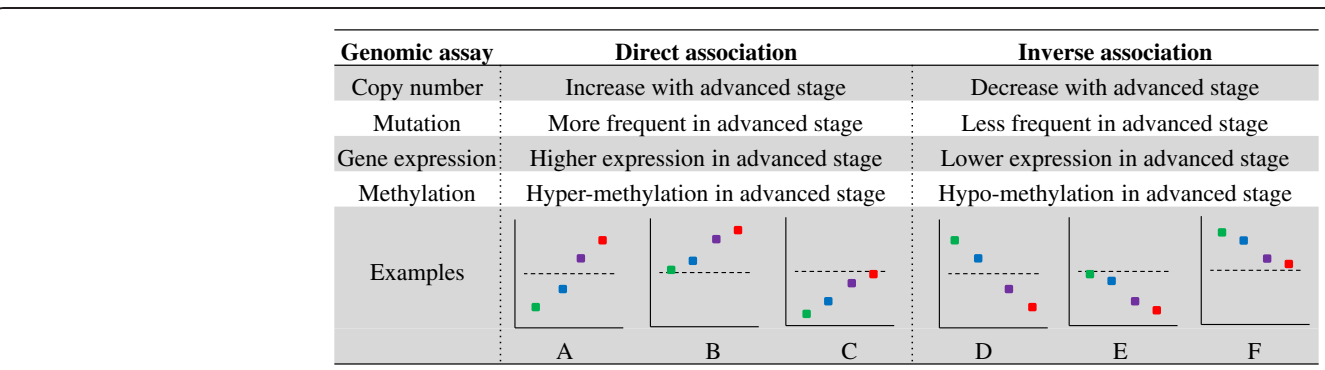

Figure 2 The interpretation of elastic net association for the comparison of low versus advanced clinical stage colorectal cancer. This figure shows comparison of lower versus higher advanced stage in direct/inverse association. The Examples row shows clinical stage I (green), II (blue), III (purple) and IV (red) respectively. The x axis represents clinical stage while y axis represents genomic changes. Dotted lines imply normal status.

we examined two aspects of its performance: i) selection of genes that are known to be associated with a clinical phenotype and ii) combination of heterogeneous genomic data from different assays. First, we applied elasticnet on a data set in which the true features and clinical associations are known. We generated four additional synthetic genes and their corresponding genomic features that are associated with clinical stage; values were generated for copy number alterations, mutation, methylation, and gene expression that were primarily present in stage III and IV CRC (Additional file 7: Figure S2). These synthetic data were appended to the fully integrated CRC data matrix. In the case of the mutation feature, we increased the frequency of mutation in each stage incrementally. Our elastic net analysis correctly identified all four synthetic genomic features and their association with clinical stages of CRC as top candidates.

We applied elastic-net regularized regression method to a TCGA study of glioblastoma (GBM) [31]. Using the same reported mutation and clinical outcome data used in the original publication reporting the TCGA GBM data, we identified $I D H 1$ to be associated with the days to death outcome. The TCGA GBM study did not identify mutations in $I D H 1$ as being clinically relevant but more recently, other studies have identified them to be indicators of poor prognosis [32].

Second, we examined the effects of variable value scales for any genomic feature occurring with the integrated CRC heterogeneous genomic data. On the integrated genomic data matrix, we increased each value 100 fold and determined any variation in the final results after elasticnet analysis. This multiplication of assay values transformed the scale of each genomic feature. Elastic-net analysis was run on this modified data set. The list of selected genes was not significantly altered.

\section{Genomic features and genes associated with MSI}

MSI is a molecular phenotype associated with loss of DNA mismatch repair function. In MSI-positive CRCs, there is a substantial increase in mutations occurring in microsatellite sequences within the coding region of critical cancer genes. The genetic basis of MSI-positive CRCs has been extensively studied and the identification of MSI mutations has revealed critical cancer genes. We conducted a supervised analysis with MSI status. We determined whether our approach could identify the important cancer genes involved in MSI-positive CRC that are already known from prior studies.

Elastic-net analysis of mutation data with MSI status identified 21 genes including TGFBR2, CASP8, and $A C V R 2 A$ while the analysis of methylation data listed 30 genes such as MLH1, FLVCR2, and EFNA1 (Table 1). The TGFBR2 gene encodes a receptor for the TGF- $\beta$ pathway and is a known cancer driver gene in MSIpositive CRC. This gene has a homopolymer $(\mathrm{A})_{10}$ tract in exon 4 which is mutation hotspot in MSI-positive CRC. This particular deletion markedly reduces mRNA levels, presumably due to nonsense-mediated decay [33]. The silencing of $M L H 1$ by hypermethylation results in the loss of DNA mismatch repair (MMR) activity and

Table 1 Top 10 genes associated with MSI

\begin{tabular}{ccccccc}
\hline & \multicolumn{3}{c}{ Mutation alone } & \multicolumn{2}{c}{ Methylation alone } & \multicolumn{2}{c}{ Integrated data } \\
\hline Rank & Gene & Sign & Gene & Sign & Gene & Sign \\
1 & TGFBR2 & $\uparrow$ & MLH1 & $\uparrow$ & MLH1 (methyl) & $\uparrow$ \\
2 & CASP8 & $\uparrow$ & FLVCR2 & $\uparrow$ & PDE6B (mut) & $\uparrow$ \\
3 & ACVR2A & $\uparrow$ & EFNA1 & $\downarrow$ & POLR1D (mut) & $\uparrow$ \\
4 & MSH3 & $\uparrow$ & CDK12 & $\uparrow$ & FLVCR2 (methyl) & $\uparrow$ \\
5 & MAP3K6 & $\uparrow$ & HOXC11 & $\downarrow$ & DAK (mRNA) & $\uparrow$ \\
6 & GATA2 & $\uparrow$ & ZNF318 & $\uparrow$ & TGFBR2 (mut) & $\uparrow$ \\
7 & MACF1 & $\uparrow$ & SEPT5 & $\uparrow$ & SEPT5 (methyl) & $\uparrow$ \\
8 & BRAF & $\uparrow$ & MIER3 & $\uparrow$ & EFNA1 (methyl) & $\downarrow$ \\
9 & GPHN & $\uparrow$ & ELK4 & $\downarrow$ & MLH1 (mRNA) & $\downarrow$ \\
10 & MAEA & $\uparrow$ & SDHD & $\downarrow$ & STK32B (mut) & $\uparrow$ \\
\hline
\end{tabular}

Gene ranking is based on the regression coefficient (see Methods). Genomic assay and associated feature is indicated in parentheses for integrative analysis of the combined genomic data. The symbol $\uparrow$ indicates increased frequency (mutation) or level (methylation) in MSI. The symbol $\downarrow$ indicates decreased frequency (mutation) or level (methylation, gene expression) in MSI. 
methylation of $M L H 1$ is frequently observed in sporadic MSI-positive CRC [34]. Our integrative analysis identified the methylation of $M L H 1$ as the top ranked MSIassociated candidate and the mutation of TGFBR2 as 6th top candidate among a total of 64 selected features (Table 1). In addition, the 9th ranked genomic feature was lower gene expression of $M L H 1$ as one would expect in the context of $M L H 1$ hypermethylation. According to TCGA analysis (January 2013), their coefficient between methylation and mRNA expression was -0.556 with $\mathrm{p}$ value of 4.43E-04. We also identified that a lack of copy number alterations in SMAD4 associated with MSI. This inverse relationship is consistent with the fact that MSI-positive mutations demonstrate a significantly lower level of copy number alterations affecting cancer genes. Overall, our results demonstrate the success of our elastic-net based analysis in identifying the MSIassociated genes. Additional file 8: Table S5 lists the full list of identified genes associated with MSI status using separate data sets.

\section{Identification of genes associated with advanced clinical with integrated analysis}

As shown in Figure 3A, the elastic-net algorithm identified genes across the different platforms that showed and association with clinical stage (e.g. Stage I, II, III, and IV). As shown in Figure 3B, 158 genomic features were selected for their association with stage III and IV CRC. We determined the ranks of these genes based on their absolute value of regression coefficient at the minimum mean squared error (MSE) determined by 10 -fold cross validation. The data and analysis results are available at open access website (http://genomeportal.stanford.edu/tcga-crc).

Overall, 158 genomic features associated with 143 genes delineated advanced clinical stage CRC. Fifteen genes demonstrate multiple genomic features (Additional file 9: Table S6). Methylation of the gene FOXP4 was the top ranked genomic feature that has the largest absolute value of regression coefficient. Since FOXP4 has a negative coefficient, it implies that FOXP4 is less methylated in advanced CRC. Only one gene, WRN, had three selected genomic features (copy number, mRNA, and methylation) while 13 genes had two genomic features. Other top ranked signatures such as REEP5, PDK4 and OR51E2 show higher gene expression in advanced stage $C R C$.

Among the 158 genomics features associated with advanced clinical stage, $45.5 \%$ are methylation, $16.8 \%$ are mutations, $31.8 \%$ are gene expression related and $8.4 \%$ are copy number alterations (Figure 3C). Gene expression features are the most common among the top 15 ranked features (12 out of top 15). An inverse association $(58.2 \%)$ is more common than direct association (41.8\%) although copy number and gene expression had similar frequency between inverse and direct association. For example, a higher portion of inverse association in methylation indicates hypermethylation in advanced
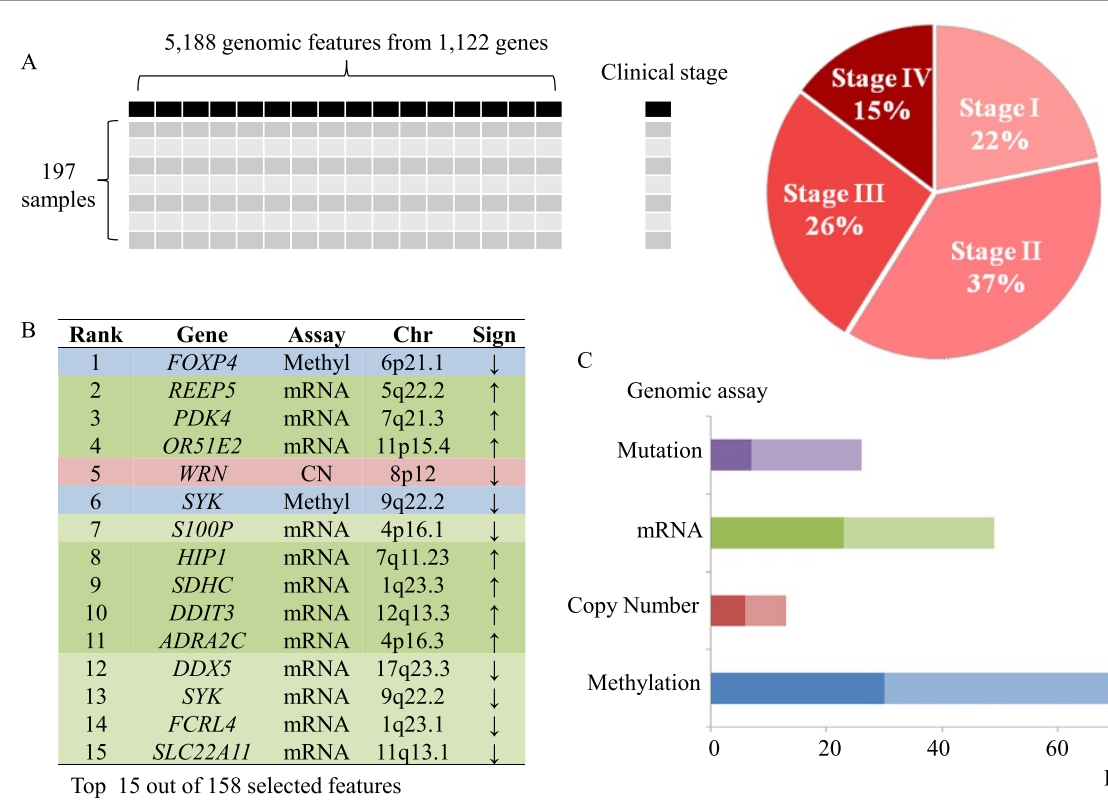

$\mathrm{C}$

Genomic assay

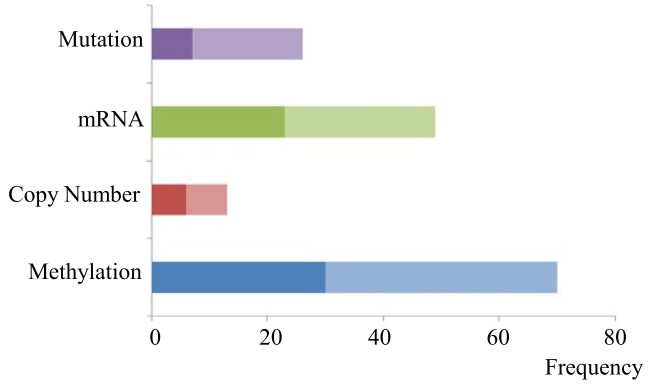

Figure 3 Model for prediction of clinical stage using regularized regression. (A) We used an integrative matrix of 5,188 genomic features from 1,122 genes in order to predict clinical stage using elastic net regularized regression. (B) The top 15 features ranked by coefficient in the model are shown. (C) Methylation features dominate the predictor set, but gene expression, copy number alteration and mutation features also play a critical role. The darker color indicates the direct association while the lighter color represents the inverse association. 
stage; as a result more genes will have lower expression in advanced stage disease.

\section{Identification of genes associated with advanced clinical stage using individual genomic feature data sets}

We ran the same elastic net regression analysis using individual genomic feature data sets (e.g. mutations alone, copy number alterations alone, etc.) compared to clinical stage (Figure 4). Elastic-net identified genomic features associated with advanced clinical stage CRC: this includes nine copy number aberrations, seven mutations, 65 gene expression changes, and 33 methylation events affecting specific genes (Additional file 9: Table S6). Only eight genes were identified with multiple genomic features: WRN, SYK, MGMT, CAPSL, ADRA2C, GNAS, IOP5 and SEMA3B. The other 96 genes were associated with only an individual genomic assay.

Our results overlapped with some of the findings of the recently published TCGA CRC study (Figure 4). The TCGA study used each genomic data set separately (e.g. mutations alone, etc.) without considering the behavior of other genetic aberration features. For their study, they determined the association between specific genetic aberrations with a number of clinical parameters including clinical stage by Fisher's test $(\mathrm{p}<0.01)$. Fifteen methylation features (out of 33), 7 gene expression features (out of 65), and 1 mutation feature (out of 7) in our study were shown to have the significant and overlapping association with clinical stage in TCGA study. Unlike our study, the original TCGA analysis of copy number alterations list large genomic intervals with multiple genes and did not identify specific genes associated with clinical stage.

We observed several differences between the integrated data set incorporating all genomic features versus an analysis of individual genomic feature data set (e.g. mutations alone, copy number alone, etc.). The integrative analysis had a better predictive power based on the mean squared error (Table 2). Likewise, different genomic features were selected from both approaches. Seventyseven out of 142 genes from integrative analysis were not listed in any separate assay analysis. This indicates these features are predictive only in the context of analyzing other genomic features representing different classes of genetic aberrations. However, the top ranked genes were quite consistent between two approaches. Seventeen of the 20 genes in integrative analysis were also identified in our analysis of the individual data sets. From the analysis of individual genomic platform data sets, the relative rankings of genes also changed compared to the fully integrated analysis. For example, when analyzing only the methylation data, the top ranked gene, WRN, dropped to a rank of 8th while the 2nd highest ranked gene, FOXP4, was the top ranked gene.

\section{Candidate genes delineating advanced CRC ranked by multi-genomic feature score}

We used our scoring scheme to prioritize the candidates (see Methods). As we noted previously, the integrative genomic analysis obtained total of 142 unique genes (Table 3, Additional file 9: Table S6) with 158 genomic features indicative of different genetic aberrations. In terms of their annotation, 56 genes are annotated in COSMIC cancer genes, two genes are MSI targets and 95 genes are reported as significant in the TCGA CRC study. Thirteen genes were listed as cancer genes in both COSMIC and the TCGA CRC study. Fourteen genes had genomic features from more than one assay. Generally, the top ten ranked genes all had multiple genomic features associated with advanced clinical stage.

WRN was the top ranked gene associated with advanced clinical stage CRC; WRN was implicated by multiple

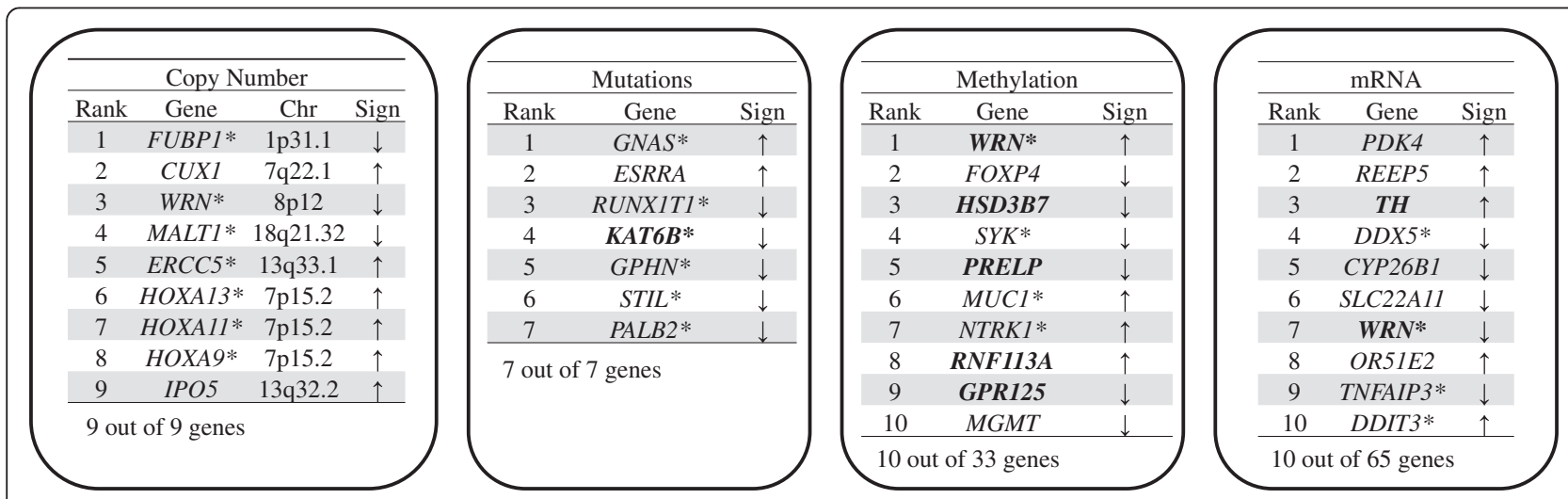

Figure 4 Prediction of clinical stage from individual genomic assay data sets. Using individual data from separate genomic assays, we conducted a supervised analysis with clinical stage. Some of these results agree with previous analysis by the TCGA; all of the features shown in bold to be associated significantly ( $p$ value $<0.01$ ) with clinical stage according to TCGA study. All association signs are identical between our analysis and TCGA analysis. A star symbol indicates cancer genes annotated by COSMIC. 
genomic features - copy number alterations, methylation, and gene expression. The WRN gene demonstrates hyper methylation $(\uparrow)$, lower copy number $(\downarrow)$ and decreased mRNA expression $(\downarrow)$ in stages III and IV (Figure 5). Overall, these different genomic features suggest that the expression of WRN is decreased in advanced clinical stage CRC. To determine the effect of other genes in proximity to WRN at locus $8 \mathrm{p} 12$, we appended the genomic features from adjacent genes neighboring WRN to the integrated data matrix. This included 13 genes telomeric and 6 genes centromeric to the 8p12 locus of WRN. Afterwards, we conducted elastic-net analysis of the original matrix. The analysis continued to identify WRN as the leading candidate gene even when taking into account the adjacent genes. The frequent deletion and methylation of WRN in advanced/CpG island methylation phenotype (CIMP) CRC has been reported [35-37]. This is external data supporting WRN's potential role in CRC.

$S Y K$ had the second highest score by mRNA expression and methylation feature. Given the central role of $S Y K$ in transferring activated immunoreceptors within B-cells, lower $S Y K$ gene expression may be involved in dampening the immune response against cancer cells. Furthermore, this gene may have a potential role in the development of human breast carcinomas [38].

We assessed the robustness of the top ranked candidates list by nonparametric bootstrap analysis (see Methods). With a 3,000 bootstrap resampling, we found that the top 25 genomic features from our initial analysis were selected repeatedly from the complete integrated genomic data set (Additional files 6: Table S4). For example, elastic-net analysis of a 3,000 bootstrap resampling data set identified gene expression of PDK 2,361 times (78.7\% among 3,000 bootstrap analysis). The WRN copy number and methylation features were identified 2,097 times (69.9\%) and 1,710 time $(57.0 \%)$ respectively. When we conducted an independent elastic net analysis to the separate individual platform genomic data sets with 3,000 bootstrap resampling, WRN was again frequently identified; 2,718 times (90.6\%) in copy number alone, 2,511 times $(83.7 \%)$ in methylation alone, and 1,749 times (58.3\%) in mRNA expression alone.

\section{Table 2 The mean squared errors (MSE) of 10 fold cross validation from Elastic-net of genomics data against clinical stages of CRC}

\begin{tabular}{lc}
\hline Genomics & MSE \\
\hline Integrative genomics & 0.78224 \\
Copy Number alone & 0.96308 \\
Mutation alone & 0.97295 \\
Methylation alone & 0.91856 \\
Gene expression alone & 0.79906 \\
\hline
\end{tabular}

Integrative analysis - the combined genomic data - showed the least MSE compared to separate genomics analysis. Therefore, the improvement was observed in integration of genomics data.
We examined the characteristics of multiple genomic features among the 14 top ranked genes associated with advanced stage clinical disease. The combination of gene expression and methylation implicate seven genes; this is the most frequent combination of features that came from our analysis. Three genes were selected with both gene expression and copy number. This suggests that methylation has a more significant influence on gene expression compared to copy number changes. For example, two genes have hypo-methylation and higher gene expression. Copy number changes are directly associated with gene expression in all three cases. In addition, we also observed the combination of copy number - methylation (1 case), mutation - methylation (3 cases), and gene expression - mutation (2 cases).

\section{Genomic features associated with individual parameters of clinical stage}

We conducted a separate supervised analysis using the individual parameters of TNM clinical stage criteria (Figure 6). As noted, $\mathrm{T}$ is assigned by extent of tumor invasion, $\mathrm{N}$ represents regional lymph node cancer involvement and $M$ represents the presence of metastatic cancer in other organs. Elastic-net identifies seven genomic features associated with higher level $\mathrm{T}$ status while 78 genomic features including ones that affected WRN are associated with $\mathrm{N}$ status (Additional file 10: Table S7). Interestingly, the three top-ranked candidates (WRN, SYK and DDX5) based on our supervised analysis of clinical stage are also associated with $\mathrm{N}$ status. This result indicates that lymph node metastasis is critical to advanced stage compared to $\mathrm{T}$ status, which is expected by AJCC staging method. There are no genes that delineated $M$ status independently. The smaller number of stage IV samples (15\%) may have affected our sensitivity for identifying genes associated with distant metastasis in other organs.

Each clinical parameter has its own unique distribution of genomic features (Figure 7A). When considering lymph node status (e.g. N), copy number alterations were most common genomic feature identified while methylation features were most common in the prediction of tumor invasiveness (e.g. T). As one may expect, mutations were relatively common feature for MSI status compared to other clinical parameters. Inverse associations are dominant in tumor invasiveness $(\mathrm{T})$ while direct associations were predominant in lymph node $(\mathrm{N})$ status and MSI (Figure 7B). The frequent direct association in $\mathrm{N}$ status and MSI implies that the gain or higher expression genetic aberrations occur more frequently in advanced versus early clinical stage. Dominant inverse association in $\mathrm{T}$ status and clinical stage suggests that loss of or lower expression of genes is a more frequent in advanced stage clinical disease. However, all 25 
Table 3 Top 25 candidates associated with advanced clinical stage

\begin{tabular}{|c|c|c|c|c|c|c|}
\hline & Gene & & & Elastic-net $\mathrm{F}$ & & \\
\hline Gene & Chr & Score & Copy Number & Gene Expression & Methylation & Mutation \\
\hline$W R N^{*}$ & $8 p 12$ & 27.03 & $5 \downarrow$ & $106 \downarrow$ & $29 \uparrow$ & \\
\hline$S Y K^{*}$ & $9 q 22.2$ & 24.09 & & $13 \downarrow$ & $6 \downarrow$ & \\
\hline$D D \times 5^{*}$ & $17 q 23.3$ & 18.94 & $70 \downarrow$ & $12 \downarrow$ & & \\
\hline$A D R A 2 C$ & $4 p 16.3$ & 18.13 & & $11 \uparrow$ & $81 \downarrow$ & \\
\hline GNAS* & $20 q 13.32$ & 16.9 & & & $39 \downarrow$ & $68 \uparrow$ \\
\hline SEMA3B & $3 p 21.31$ & 16.66 & & $82 \downarrow$ & $28 \downarrow$ & \\
\hline HSD17B2 & $16 q 23.3$ & 14.45 & $17 \uparrow$ & $120 \uparrow$ & & \\
\hline TTN & $2 q 31.2$ & 13.72 & & $80 \downarrow$ & & $66 \downarrow$ \\
\hline FHIT* & $3 p 14.2$ & 13.56 & & & $71 \uparrow$ & $77 \downarrow$ \\
\hline HIST1H4I* & $6 \mathrm{p} 22.1$ & 12.82 & & $35 \uparrow$ & $122 \downarrow$ & \\
\hline FOXP4 & $6 \mathrm{p} 21.1$ & 12.74 & & & $1 \downarrow$ & \\
\hline REEP5 & $5 q 22.2$ & 12.66 & & $2 \uparrow$ & & \\
\hline PDK4 & $7 q 21.3$ & 12.58 & & $3 \uparrow$ & & \\
\hline OR51E2 & $11 \mathrm{p} 15.4$ & 12.49 & & $4 \uparrow$ & & \\
\hline S100P & $4 \mathrm{p} 16.1$ & 12.25 & & $7 \downarrow$ & & \\
\hline$H I P 1^{*}$ & $7 q 11.23$ & 12.17 & & $8 \uparrow$ & & \\
\hline ZNF570 & $19 q 13.12$ & 12.09 & & $50 \uparrow$ & & $116 \downarrow$ \\
\hline$S D H C^{*}$ & $1 \mathrm{q} 23.3$ & 12.09 & & $9 \uparrow$ & & \\
\hline DDIT3* & $12 q 13.3$ & 12 & & $10 \uparrow$ & & \\
\hline CRTC1* & 19p13.11 & 11.92 & & & $130 \uparrow$ & $38 \downarrow$ \\
\hline FCRL4* & $1 q 23.1$ & 11.84 & & $14 \downarrow$ & $155 \downarrow$ & \\
\hline SLC22A11 & $11 q 13.1$ & 11.6 & & $15 \downarrow$ & & \\
\hline FLT1 & $13 q 12.2$ & 11.51 & & $16 \uparrow$ & & \\
\hline CYP26B1 & $2 \mathrm{p} 13.2$ & 11.35 & & $18 \downarrow$ & & \\
\hline RNF113A & $X q 24$ & 11.27 & & & $19 \uparrow$ & \\
\hline
\end{tabular}

We ranked the genes by selected feature scoring (see Method for scoring scheme). This scoring scheme selects genes that are supported by multiple and/or highly ranked genomic features. Elastic-net feature rank indicates the rank of individual assay features, where $\uparrow$ indicates a direct association with the feature value and $\downarrow$ indicates inverse association with the feature value. COSMIC-annotated cancer genes are marked by *.

genomic features common in clinical stage and $\mathrm{N}$ status had identical association directions; 14 with direct associations and 11 with inverse associations.

If one includes the results from all of the tested clinical parameters, a total of 237 genes are identified. There are no genes selected independently that predicted all four clinical parameters. The majority of genes (80.5\%) are associated with a single clinical parameter. Three genes were identified repeatedly among 3 different clinical parameters: BCL2, TRERF1, and MGMT. Forty-three genes were associated with two different clinical parameters.

\section{Identification of miRNAs associated with CRC clinical stage}

As noted previously miRNA data was analyzed separately because of extensive gaps in the data and the general ambiguity about which genes are regulated by
miRNAs. We ran an elastic net regression on miRNA data separately in comparison with clinical stage. We used the same 197 CRC samples as the integrative genomic analysis. There are two types of genomics data for miRNA; copy number alterations and expression. The copy number data included 2,260 miRNAs while the expression data covered 420 miRNAs. First, we integrated both miRNA copy number and expression into a single matrix. However, this combined data analysis did not produce any candidates associated with advanced clinical stage. Second, we ran the elastic net regression separately on copy number and expression data. From the expression data set, our analysis identifies $33 \mathrm{miR}$ NAs associated with clinical stage (Additional file 11: Table S8). The top candidate, MIR21, was suggested as a potential diagnostic marker of colorectal cancer [39] and involved in tumor growth in breast cancer [40]. 


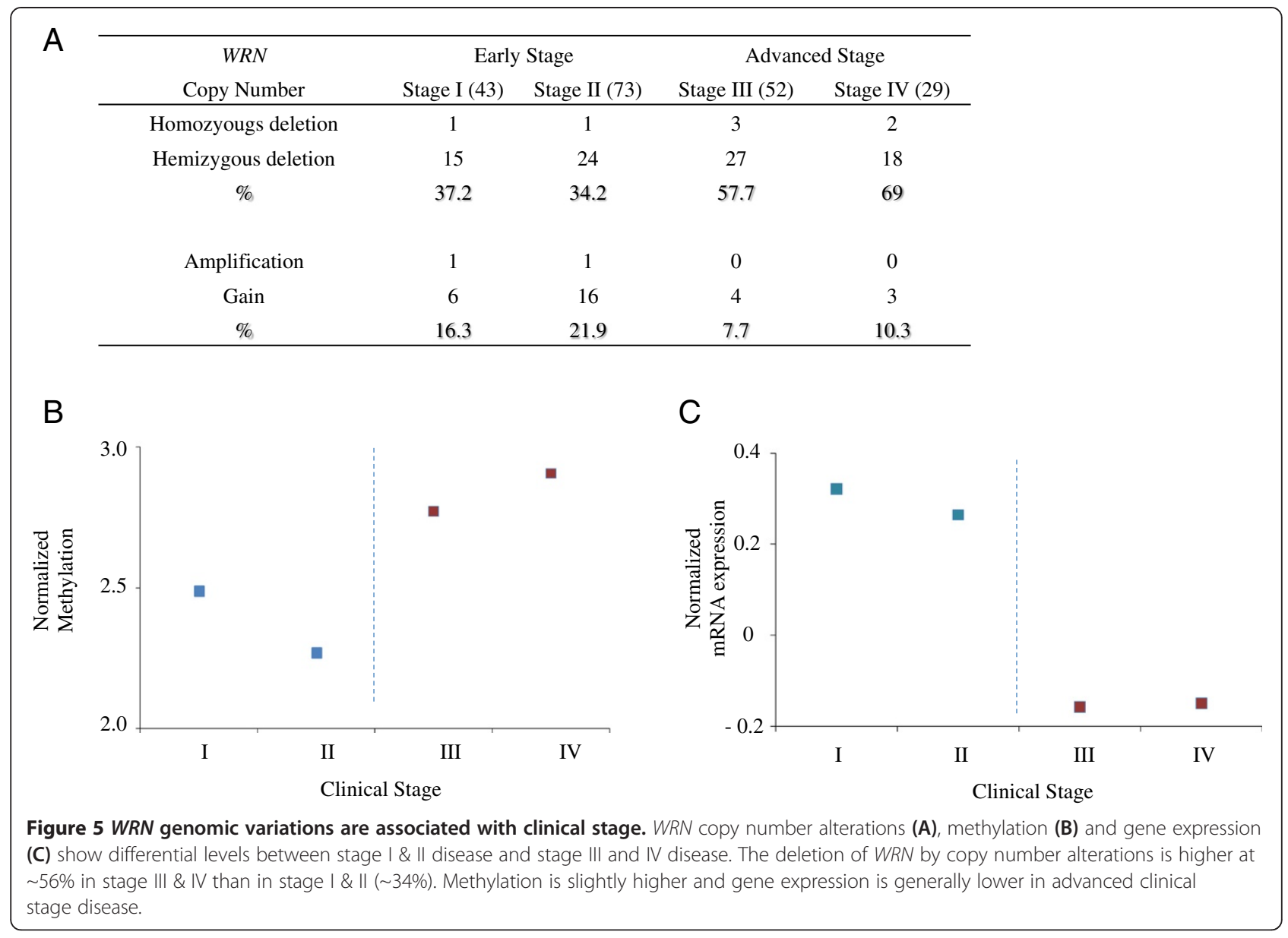

Interestingly, MIR21 is located at $17 \mathrm{q} 23.1$ in relative proximity to the top 3rd stage associated gene, DDX3 (17q23.3). None of 33 miRNAs are located in loci for the other top candidate genes such as $8 \mathrm{p} 12$ (WRN), 9q22 (SYK), or 4p16 (ADRA2C).

\section{Discussion}

We conducted a regularized elastic-net regression analysis of heterogeneous genomic data that encompassed multiple classes of genetic aberrations (i.e. genomic features) in colorectal cancer. We identified 158 genomic features associated with advanced clinical stage with 10 -fold cross validation (Figure 3 ) and ranked these genes based on by their regression coefficient and level of support from multiple assay modalities (Table 3). Our integrative analysis approach provides a better picture about the distribution of genomic features associated with clinical parameters, which cannot be obtained by separately analyzing individual classes of genetic aberrations such as mutations alone (Figure 7). For example, our integrative analysis identified specific mutations that delineate clinical stage in the context of overlapping genomic features. This was not possible when analyzing mutations alone without the genomic context of other genetic aberrations. We also demonstrated that the predictive power was relatively improved using our integrative analysis approach (Table 2) as several other studies have demonstrated previously [24-29]. Despite of slight increase in predictive power, integrative analysis enabled us to identify critical genes that are supported by more than one genomic biomarker. Several top candidates such as GNAS (supported by methylation and mutation) and FHIT (supported by methylation and mutation) would not be detected if analysis was solely based on gene expression data.

The leading candidate, WRN, showed the higher number of genomic features that can delineate advanced clinical stage CRC (Figure 5). WRN is a known tumor suppressor. Hereditary germ line mutations in the WRN gene cause the adult-progerioid genetic disorder known as Werner's syndrome (WS). This disorder is associated with an increased risk of cancer [41-43]. The increased risk of cancer in individuals with WS has established the role of WRN as a tumor suppressor gene. However, genetic aberrations in WRN have never been described in sporadic CRC development. WRN is a human RecQ 


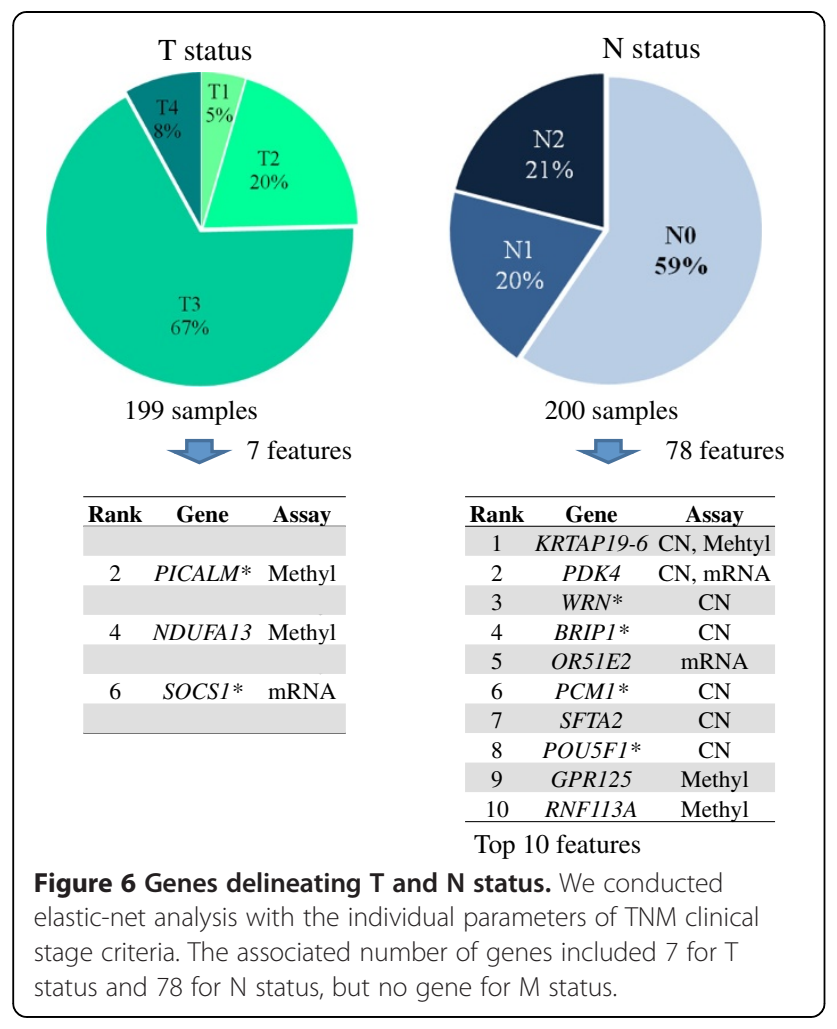

Figure 6 Genes delineating $\mathrm{T}$ and $\mathbf{N}$ status. We conducted elastic-net analysis with the individual parameters of TNM clinical status and 78 for $\mathrm{N}$ status, but no gene for $\mathrm{M}$ status.
DNA helicase family and has both the characteristic 3' to $5^{\prime}$ helicase activity and also a $3^{\prime}$ to $5^{\prime}$ exonuclease activity. Generally, we observed the WRN was subject to hemizygous gene loss, a category of genetic aberrations that are increasingly being scrutinized for a specific role in cancer development. Solimini et al. recently provided evidence that the gene dosage changes as result of hemizygous deletions play an important role in tumor progression [44]. We are pursuing translational validation studies and further experiments to address the role of WRN in advanced CRC.

The results from analysis of the individual clinical parameters of the TMN criteria provided us with additional biological insight. There was significant overlap between genes associated with advanced clinical stage and $\mathrm{N}$ status, more so than any other comparison among the various clinical parameters we examined. This may be an indication that genes associated with lymph node invasion are relatively critical for delineating advanced stage of CRC compared to other parameters such as tumor invasiveness level. This conclusion is supported by the fact that lymph node status remains the most important and reproducible prognostic clinical parameter for colon cancer [45]. Interestingly, we did not identify a gene specific for distant organ metastasis. This may be related to the smaller number of stage IV CRCs in early releases of the TCGA data set or the potential greater role of lymph node metastasis as an early indicator of

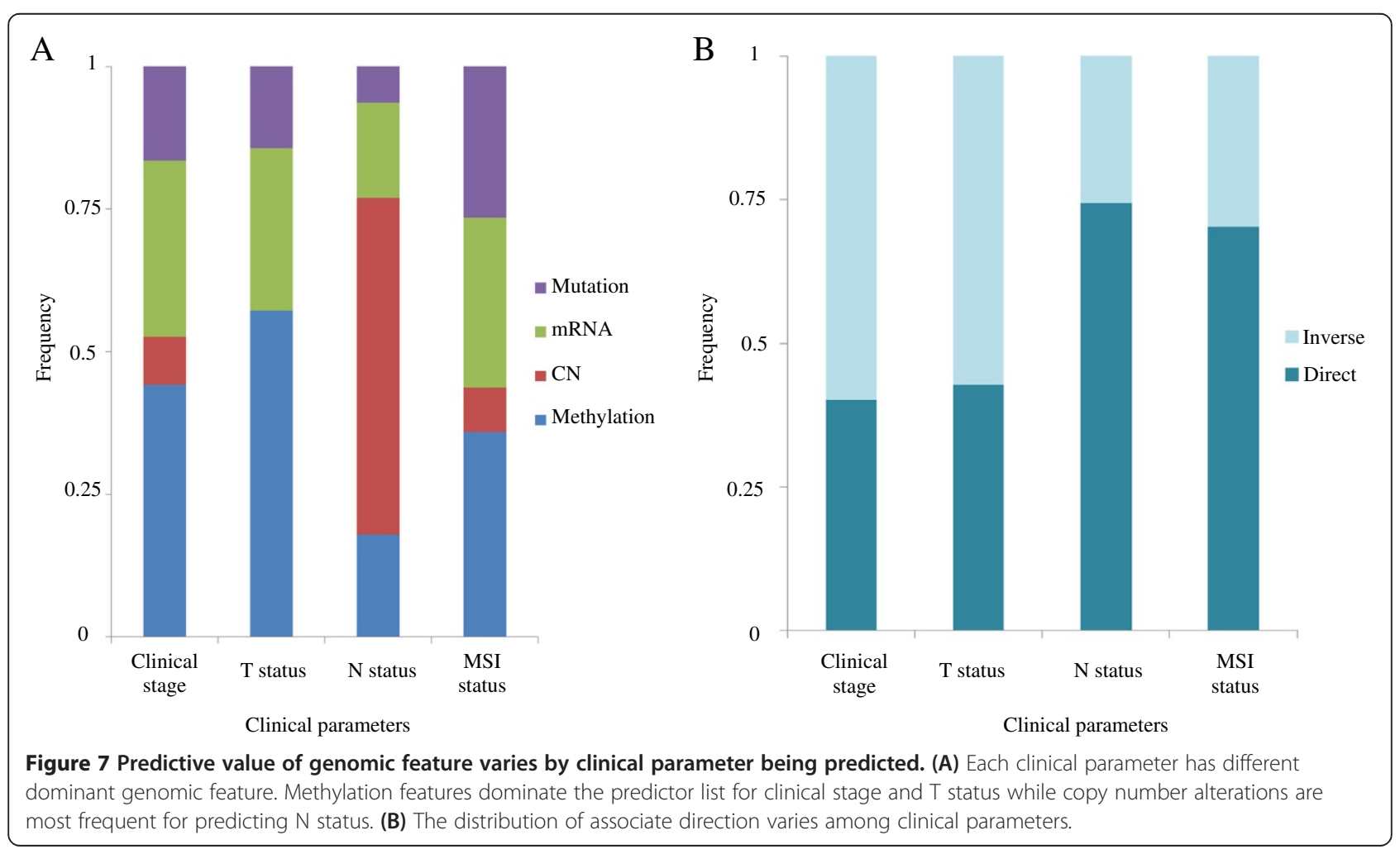


metastatic progression. By grouping stage III and IV patients as advanced disease, we improve the sample size at the risk of increasing within-group heterogeneity. As the TCGA CRC data set matures, we will have opportunity to analyze a large data set for associations.

We evaluated the performance of elastic-net on the large genomic data set with clinical information by using control data sets. Our analysis successfully identified the clinically relevant genomic signatures in three cases: i) Identification of synthetic genes that have stage-associated genomic features, ii) Identification of the $I D H 1$ gene from GBM mutation data and iii) identification of methylation on $M L H 1$ and mutations on TGFBR2 for MSI status of CRC. In the case of the CRC stage association, some of the selected genomic features for CRC stage from each genomic assay were consistent with the results from the TCGA CRC study (Figure 4). Our study supports the overall reliability of the candidates associated with clinical stage from elastic-net.

Interestingly, cancer genes (e.g. TP53, KRAS, APC) with the highest frequency of genetic aberrations were not among the genes identified in delineating advanced clinical stage of CRC. These cancer driver genes were mutated frequently across all clinical stages. For instance, $A P C$ was mutated in $90 \%, 74 \%, 77 \%$, and $93 \%$ of stage $\mathrm{I}$, II, III and IV respectively. Therefore, our interpretation is that while these cancer drivers play a critical role in the initial neoplastic development and maintenance, they play lesser role for influencing metastatic progression and advanced clinical stage.

Our study is distinct and novel compared to the TCGA CRC study; unlike TCGA, we considered four major features of genetic aberrations simultaneously rather than specific genetic aberrations in isolation. As we demonstrate, this improves the performance of our approach; it leads us to some gene candidates delineating advanced clinical stage not previously recognized. Integration of different genomic data gives us more informative results as we have shown it in this study. Multiple genomic features enable us to prioritize the gene aberration for follow-up validation studies. Another feature of our study is the flexibility of our analysis approach. Using elastic net, we can readily modify our bioinformatics pipeline to consider other clinical parameters. In the future, we anticipate applying additional clinical parameters such as drug response for our analysis as these data become available.

One of the main limitations of our study is the absence of an independent data set for validation. We demonstrated the robustness of our candidates by bootstrap resampling. We also considered over 11 other genomic studies of CRC that looked at differences among early versus advanced stage disease [46]. Only two analyzed tumors from all clinical stages usually with less than 80 samples. In addition, all of these studies used older gene expression microarrays lacking the number of features see in the TCGA and thus never reached the level of comprehensive multiple-platform analysis or high genomic resolution as was conducted by the TCGA. For this analysis, the ability to integrate heterogeneous genomic features was critical and the limitations of the other data sets made them less useful for our integrated analysis. As a validation analysis with a single platform, we opted to use an independent set of 354 samples with both clinical data and copy number variation data from the expanded TCGA CRC data. This separate analysis validated $M A L T 1$ as a top hit when only considering copy number analysis.

We also considered an integrative analysis of independent CRC samples from TCGA. At the time of this study, many of the TCGA samples have not undergone analysis with all of the genomic assays. Many of the other CRC samples, outside of the ones we use, lack sufficient clinical data. In the future, with the completion of the TCGA study and adequate clinical annotation afterwards, we will use the additional data sets from independent samples from our original analysis.

To improve our analysis, we are testing methods to integrate disparate classes of data; for example, this genomic data covers a range including binary for mutation, categorical for copy number, and continuous for mRNA and methylation. One potential approach involves turning the values of every genomic assay into categorical variable by discretization. For example, GISTIC is a method for producing discrete values for discriminating significant copy number alterations. However, discretization is less applicable to continuous data sets such as gene expression and methylation. Another possibility involves converting every genomic assay feature into a continuous value. For example, we can use several programs that predict the functional impacts of mutations, thus convert binary mutation values (e.g. stop mutation, substitutions, etc.) into continuous pathogenicity values. We will test whether homogeneity in different genomic values may improve the predictive power of our analysis and improve the ranking of genes in terms of their importance biologically and clinically.

\section{Conclusions}

Leveraging the expansive and comprehensive data sets of the TCGA, we developed a robust and straightforward approach to integrate and analyze heterogeneous cancer genomic data sets. Based on a supervised comparison of clinical parameters indicative of advanced CRC, we utilized in parallel mutation, gene expression, copy number alteration and methylation data and we identify genes associated with advanced clinical stage, several of which have not been identified previously. 


\section{Additional files}

Additional file 1: Table S1. Change clinical parameters into ordinal values. Additional file 2: Table S2. The list of selected genes.

Additional file 3: Analysis scripts and commands.

Additional file 4: Figure S1. Gene expression eigen scatter plot. Additional file 5: Table S3. The list of samples with their clinical information.

Additional file 6: Table S4. Results of bootstrap analysis. Additional file 7: Figure S2. Elastic-net analysis of synthetic genetic features.

Additional file 8: Table S5. The list of genes associated with MSI.

Additional file 9: Table S6. The list of genes associated with clinical stage. Additional file 10: Table S7. The list of genes associated with T status and $\mathrm{N}$ status.

Additional file 11: Table S8. The list of micro RNAs associated with clinical stage

\section{Abbreviations}

CRC: Colorectal cancer; TCGA: The cancer genome atlas; SNP: Single nucleotide polymorphism; TGF- $\beta$ : Transforming growth factor $\beta$; miRNA: micro RNA; MSI: Microsatellite instability; GBM: Glioblastoma; MMR: Mismatch repair; MSE: Mean squared error; GDACs: Genome data analysis centers; HGNC: HUGO gene nomenclature; PCA: Principal component analysis; LASSO: Least absolute shrinkage and selection operator; WS: Werner's syndrome; COSMIC: The Catalogue of somatic mutations in cancer; RPKM: Reads per kilo base per million; T status: Extent of tumor invasion; N status: Regional lymph node cancer involvement; M status: Presence of metastatic cancer in other organs; CIMP: CpG island methylation phenotype.

\section{Competing interests}

The authors declare that they have no competing interests.

\section{Authors' contributions}

PF, HJL and HPJ designed the study. HJL and PF conducted the analysis. HPJ supervised and coordinated the overall study. All authors revised, read and approved the final manuscript.

\section{Acknowledgements}

This work was supported by the following grants from the $\mathrm{NIH}$ : P01HG000205 to PF and HPJ; 1U01CA15192001-A1 to HJL and HPJ.

1U01CA176299 to HPJ. In addition, HPJ was supported by a Research Scholar Grant, RSG-13-297-01-TBG from the American Cancer Society, the Doris Duke Clinical Foundation, the Wang Family Foundation and the Howard Hughes Medical Foundation. We acknowledge Sue Grimes and Jennifer Palm in providing support for the web implementation.

\section{Author details}

${ }^{1}$ Division of Oncology, Stanford University School of Medicine, Stanford, CA 94305, USA. ²Department of Biomedical Engineering, Worcester Polytechnic Institute, Worcester, MA 01605, USA. ${ }^{3}$ Stanford Genome Technology Center, Stanford University, Palo Alto, CA 94304, USA

Received: 19 September 2013 Accepted: 27 November 2013 Published: 5 December 2013

\section{References}

1. Siegel R, Naishadham D, Jemal A: Cancer Statistics, 2013. CA Cancer J Clin 2013, 63:11-30.

2. Vogelstein B, Kinzler KW: Cancer genes and the pathways they control. Nat Med 2004, 10:789-799.

3. Wood LD, Parsons DW, Jones S, Lin J, Sjöblom T, Leary RJ, Shen D, Boca SM, Barber T, Ptak J, Silliman N, Szabo S, Dezso Z, Ustyanksky V, Nikolskaya T, Nikolsky Y, Karchin R, Wilson PA, Kaminker JS, Zhang Z, Croshaw R, Willis J, Dawson D, Shipitsin M, Willson JKV, Sukumar S, Polyak K, Park BH,
Pethiyagoda CL, Pant PVK, et al: The genomic landscapes of human breast and colorectal cancers. Science 2007, 318:1108-1113.

4. Sjöblom T, Jones S, Wood LD, Parsons DW, Lin J, Barber TD, Mandelker D, Leary RJ, Ptak J, Silliman N, Szabo S, Buckhaults P, Farrell C, Meeh P, Markowitz SD, Willis J, Dawson D, Willson JKV, Gazdar AF, Hartigan J, Wu L, Liu C, Parmigiani G, Park BH, Bachman KE, Papadopoulos N, Vogelstein B, Kinzler KW, Velculescu VE: The consensus coding sequences of human breast and colorectal cancers. Science 2006, 314:268-274.

5. Fearon ER: Molecular genetics of colorectal cancer. Annu Rev Pathol 2011, 6:479-507.

6. Fearon ER, Vogelstein B: A genetic model for colorectal tumorigenesis. Cell 1990, 61:759-767.

7. Vogelstein B, Papadopoulos N, Velculescu VE, Zhou S, Diaz LA, Kinzler KW: Cancer genome landscapes. Science 2013, 339:1546-1558.

8. Forbes $\mathrm{S}$ a, Bindal N, Bamford S, Cole C, Kok CY, Beare D, Jia M, Shepherd R, Leung K, Menzies A, Teague JW, Campbell PJ, Stratton MR, Futreal PA: COSMIC: mining complete cancer genomes in the catalogue of somatic mutations in cancer. Nucleic Acids Res 2011, 39(Database issue):D945-D950.

9. Bienz M, Clevers H: Linking colorectal cancer to Wnt signaling. Cell 2000, 103:311-320.

10. Shirasawa S, Furuse M, Yokoyama N, Sasazuki T: Altered growth of human colon cancer cell lines disrupted at activated Ki-ras. Science 1993, 260:85-88.

11. Pasche B, Kaklamani V, Hou N, Young T, Rademaker A, Peterlongo P, Ellis N, Offit $K$, Caldes T, Reiss M, Zheng T: TGFBR $1{ }^{*} 6 A$ and cancer: a meta-analysis of 12 case-control studies. J Clin Oncol 2004, 22:756-758.

12. Mutch MG: Molecular profiling and risk stratification of adenocarcinoma of the colon. J Surg Oncol 2007, 96:693-703.

13. Muzny DM, Bainbridge MN, Chang K, Dinh HH, Drummond J a, Fowler G, Kovar CL, Lewis LR, Morgan MB, Newsham IF, Reid JG, Santibanez J, Shinbrot E, Trevino LR, Wu YQ, Wang M, Gunaratne P, Donehower L a, Creighton CJ, Wheeler D a, Gibbs R a, Lawrence MS, Voet D, Jing R, Cibulskis K, Sivachenko A, Stojanov P, McKenna A, Lander ES, Gabriel S, et al: Comprehensive molecular characterization of human colon and rectal cancer. Nature 2012, 487:330-337.

14. Shen $R$, Olshen $A B$, Ladanyi M: Integrative clustering of multiple genomic data types using a joint latent variable model with application to breast and lung cancer subtype analysis. Bioinformatics 2009, 25:2906-2912.

15. Vucic EA, Thu KL, Robison K, Rybaczyk LA, Chari R, Alvarez CE, Lam WL: Translating cancer "omics" to improved outcomes. Genome Res 2012, 22:188-195.

16. Chin L, Hahn WC, Getz G, Meyerson M: Making sense of cancer genomic data. Genes Dev 2011, 25:534-555.

17. Goldman M, Craft B, Swatloski T, Ellrott K, Cline M, Diekhans M, Ma S, Wilks C, Stuart J, Haussler D, Zhu J: The UCSC cancer genomics browser: update 2013. Nucleic Acids Res 2013, 41(Database issue):D949-D954.

18. Woerner SM, Yuan YP, Benner A, Korff S, von Knebel Doeberitz M, Bork P. SelTarbase, a database of human mononucleotide-microsatellite mutations and their potential impact to tumorigenesis and immunology. Nucleic Acids Res 2010, 38(Database issue):D682-D689.

19. Vilar E, Gruber SB: Microsatellite instability in colorectal cancer-the stable evidence. Nat Rev Clin Oncol 2010, 7:153-162.

20. Gray K, Daugherty LC, Gordon SM, Seal RL, Wright MW, Bruford E: Genenames.org: the HGNC resources in 2013. Nucleic Acids Res 2013, 41(Database issue):D545-D552.

21. I. T. Jolliffe: Principal Component Analysis (Springer Series in Statistics). 2nd edition. New York: Springer; 2002.

22. Tusher VG, Tibshirani R, Chu G: Significance analysis of microarrays applied to the ionizing radiation response. Proc Natl Acad Sci U S A 2001 98:5116-5121.

23. Zou H, Hastie T: Regularization and variable selection via the elastic net. J R Stat Soc Ser B (Statistical Methodol 2005, 67:301-320.

24. Chin K, DeVries S, Fridlyand J, Spellman PT, Roydasgupta R, Kuo W-L, Lapuk A, Neve RM, Qian Z, Ryder T, Chen F, Feiler H, Tokuyasu T, Kingsley C, Dairkee S, Meng Z, Chew K, Pinkel D, Jain A, Ljung BM, Esserman L, Albertson DG, Waldman FM, Gray JW: Genomic and transcriptional aberrations linked to breast cancer pathophysiologies. Cancer Cell 2006, 10:529-541.

25. Curtis C, Shah SP, Chin S-F, Turashvili G, Rueda OM, Dunning MJ, Speed D, Lynch AG, Samarajiwa S, Yuan Y, Graf S, Ha G, Haffari G, Bashashati A, Russell R, McKinney S, Langerod A, Green A, Provenzano E, Wishart G, Pinder S, 
Watson P, Markowetz F, Murphy L, Ellis I, Purushotham A, Borresen-Dale A-L, Brenton JD, Tavare S, Caldas $C$, et al: The genomic and transcriptomic architecture of 2,000 breast tumours reveals novel subgroups. Nature 2012, 486:346-352.

26. Shen L, Toyota M, Kondo Y, Lin E, Zhang L, Guo Y, Hernandez NS, Chen X, Ahmed S, Konishi K, Hamilton SR, Issa J-PJ: Integrated genetic and epigenetic analysis identifies three different subclasses of colon cancer. Proc Natl Acad Sci U S A 2007, 104:18654-18659.

27. Mo Q, Wang S, Seshan VE, Olshen AB, Schultz N, Sander C, Powers RS, Ladanyi M, Shen R: Pattern discovery and cancer gene identification in integrated cancer genomic data. Proc Natl Acad Sci U S A 2013, 110:4245-4250.

28. Kristensen VN, Vaske CJ, Ursini-Siegel J, Van Loo P, Nordgard SH, Sachidanandam R, Sørlie T, Wärnberg F, Haakensen VD, Helland A, Naume B, Perou CM, Haussler D, Troyanskaya OG, Børresen-Dale A-L: Integrated molecular profiles of invasive breast tumors and ductal carcinoma in situ (DCIS) reveal differential vascular and interleukin signaling. Proc Natl Acad Sci U S A 2012, 109:2802-2807.

29. Ernst J, Plasterer HL, Simon I, Bar-Joseph Z: Integrating multiple evidence sources to predict transcription factor binding in the human genome. Genome Res 2010, 20:526-536.

30. Davison AC, Hinkley DV: Bootstrap Methods and Their Application. Cambridge: Cambridge University Press; 1997.

31. Cancer T, Atlas G: Comprehensive genomic characterization defines human glioblastoma genes and core pathways. Nature 2008, 455:1061-1068.

32. Yan $H$, Parsons DW, Jin G, McLendon R, Rasheed BA, Yuan W, Kos I, Batinic-Haberle I, Jones S, Riggins GJ, Friedman H, Friedman A, Reardon D, Herndon J, Kinzler KW, Velculescu VE, Vogelstein B, Bigner DD: IDH1 and IDH2 mutations in gliomas. N Engl J Med 2009, 360:765-773.

33. Markowitz S, Wang J, Myeroff L, Parsons R, Sun L, Lutterbaugh J, Fan RS, Zborowska E, Kinzler KW, Vogelstein B: Inactivation of the type II TGF-beta receptor in colon cancer cells with microsatellite instability. Science 1995, 268:1336-1338.

34. Kane MF, Loda M, Gaida GM, Lipman J, Mishra R, Goldman H, Jessup JM, Kolodner R: Methylation of the hMLH1 promoter correlates with lack of expression of $\mathrm{hMLH} 1$ in sporadic colon tumors and mismatch repair-defective human tumor cell lines. Cancer Res 1997, 57:808-811.

35. Chughtai SA, Crundwell MC, Cruickshank NR, Affie E, Armstrong S, Knowles MA, Takle LA, Kuo M, Khan N, Phillips SM, Neoptolemos JP, Morton DG: Two novel regions of interstitial deletion on chromosome $8 \mathrm{p}$ in colorectal cancer. Oncogene 1999, 18:657-665.

36. Kawasaki T, Ohnishi M, Suemoto Y, Kirkner GJ, Liu Z, Yamamoto H, Loda M, Fuchs CS, Ogino S: WRN promoter methylation possibly connects mucinous differentiation, microsatellite instability and $\mathrm{CpG}$ island methylator phenotype in colorectal cancer. Mod Pathol 2008, 21:150-158.

37. Agrelo R, Cheng W-H, Setien F, Ropero S, Espada J, Fraga MF, Herranz M, Paz MF, Sanchez-Cespedes M, Artiga MJ, Guerrero D, Castells A, von Kobbe C, Bohr VA, Esteller M: Epigenetic inactivation of the premature aging Werner syndrome gene in human cancer. Proc Natl Acad Sci U S A 2006, 103:8822-8827.

38. Coopman PJ, Do MT, Barth M, Bowden ET, Hayes AJ, Basyuk E, Blancato JK, Vezza PR, McLeskey SW, Mangeat PH, Mueller SC: The Syk tyrosine kinase suppresses malignant growth of human breast cancer cells. Nature 2000, 406:742-747.

39. Kanaan Z, Rai SN, Eichenberger MR, Roberts H, Keskey B, Pan J, Galandiuk S: Plasma miR-21: a potential diagnostic marker of colorectal cancer. Ann Surg 2012, 256:544-551.

40. Si M-L, Zhu S, Wu H, Lu Z, Wu F: Mo Y-Y: miR-21-mediated tumor growth. Oncogene 2007, 26:2799-2803.

41. Yamamoto K, Imakiire A, Miyagawa N, Kasahara T: A report of two cases of Werner's syndrome and review of the literature. 2003, 11:224-233.

42. Hickson ID: RecQ helicases: Caretakers of the genome. Nat Rev Cancer 2003, 3:169-178.

43. Epstein CJ, Martin GM, Schultz AL, Motulsky AG: Werner's syndrome a review of its symptomatology, natural history, pathologic features, genetics and relationship to the natural aging process. Medicine (Baltimore) 1966, 45:177-221.

44. Solimini $\mathrm{NL}$, Xu Q, Mermel $\mathrm{CH}$, Liang AC, Schlabach MR, Luo J, Burrows AE, Anselmo AN, Bredemeyer AL, Li MZ, Beroukhim R, Meyerson M, Elledge SJ: Recurrent hemizygous deletions in cancers may optimize proliferative potential. Science 2012, 337:104-109.
45. Compton CC, Fielding LP, Burgart $L$, Conley B, Cooper HS, Hamilton SR, Hammond MEH, Henson DE, Hutter RVP, Nagle RB, Nielsen ML, Sargent DJ Taylor CR, Welton M, Willett C: Prognostic factors in colorectal cancer. Arch Pathol Lab Med 2000, 124:979-994.

46. Shibayama M, Maak M, Nitsche U, Gotoh K, Rosenberg R, Janssen K-P: Prediction of metastasis and recurrence in colorectal cancer based on gene expression analysis: Ready for the clinic? Cancers (Basel) 2011, 3:2858-2869.

doi:10.1186/1755-8794-6-54

Cite this article as: Lee et al:: Systematic genomic identification of colorectal cancer genes delineating advanced from early clinical stage and metastasis. BMC Medical Genomics 2013 6:54.

\section{Submit your next manuscript to BioMed Central and take full advantage of:}

- Convenient online submission

- Thorough peer review

- No space constraints or color figure charges

- Immediate publication on acceptance

- Inclusion in PubMed, CAS, Scopus and Google Scholar

- Research which is freely available for redistribution

Submit your manuscript at www.biomedcentral.com/submit
C Biomed Central 